\title{
A NOTE ON SOBOLEV TYPE INEQUALITIES ON GRAPHS WITH POLYNOMIAL VOLUME GROWTH
}

\author{
LI CHEN
}

\begin{abstract}
We prove the generalized $L^{p}$-Poincaré inequalities and Sobolev type inequalities on graphs with polynomial volume growth. They are optimal on Vicsek graphs.
\end{abstract}

\section{INTRODUCTION}

The classical Sobolev inequalities play a crucial role in analysis and partial differential equations in Euclidean spaces. In many different settings, Sobolev inequalities have been deeply explored and they are extremely flexible and useful. For a nice introduction of previous work, we refer to the survey by L. SaloffCoste [15], see also the comprehensive book [14].

In this note we work on a scale of Sobolev type inequalities of great generality in the setting of graphs, which was first introduced by T. Coulhon in [7] on manifolds and graphs. Let $D>1$ and $1 \leq p \leq \infty$, consider on an infinite graph $\Gamma$ the following scale of inequalities

$$
\|f\|_{p} \leq|\Omega|^{1 / D}\||\nabla f|\|_{p}
$$

for any finite subset $\Omega$ of $\Gamma$ and any function $f$ supported in $\Omega$, where $|\Omega|$ denotes the measure of $\Omega$.

The above inequalities are also quoted as Faber-Krahn type inequality (see for instance [7, page 88]). When $p<D$, the inequality (1.1) is equivalent to the graph analogue of classical Sobolev inequalities. For more interpretations from either geometric or analytic aspects, we refer to [7, 6, 4]. Of the same spirit, a more general version of (1.1) was also studied in the context of manifolds and graphs in $[6,8,10]$, that is,

$$
\|f\|_{p} \leq \varphi(|\Omega|)\||\nabla f|\|_{p},
$$

where $\varphi$ is a non-decreasing function on $\mathbb{R}^{+}$to itself.

Volume lower bounds are important implications of the above two scales. Indeed, (1.1) implies that $|B(x, n)| \geq C n^{D}$. For the more general scale, if $\varphi$ is unbounded, then (1.2) for $p=\infty$ is equivalent to $|B(x, n)| \geq C \varphi^{-1}(n)$, where $\varphi^{-1}$ is the reverse function of $\varphi$ (see [8, Proposition 2.2] or [6]). The reverse implication is well studied under the assumption of pseudo-Poincaré inequality

$$
\left\|f-f_{n}\right\|_{p} \leq C n\||\nabla f|\|_{p},
$$

where $f_{n}(x)=\frac{1}{|B(x, n)|} \sum_{y \in B(x, n)} f(y) \mu(y)$, where $\mu$ is the measure on $\Gamma$. In fact, this allows the scale to go down from $\infty$ to $p$, see [8, Proposition 2.6]. Compared with the Poincaré inequality

$$
\left\|f-f_{B(x, n)}\right\|_{L^{p}(B(x, n))} \leq C n\||\nabla f|\|_{L^{p}(B(x, 2 n))},
$$

the pseudo-Poincaré inequality may hold for more general situations, for instance on all unimodular Lie groups (see [8]). As is well-known, the Poincaré inequality and the volume doubling property imply the pseudo-Poincaré inequality (see for example [13], [14]). On graphs both the Poincaré and pseudo-Poincaré inequalities are very restrictive properties. However, under assumptions of volume growth, one can obtain analogues of the $L^{1}$ and $L^{2}$-Poincaré inequalities, see [11, Section 5].

In this note, our goals are to generalize the results in [11] to the case $1<p<\infty$ on graphs with polynomial volume growth and hence to study the scale of Sobolev type inequalities in form of (1.2). We are particularly interested in explicit examples of Vicsek graphs (see [2, 3]). 
In the following we introduce the setting of graphs, mainly following the notation in [4]. Let $\Gamma=(V, E)$ be a connected undirected infinite graph with the set of vertices $V$ and the set of edges $E$. Endow $\Gamma$ with a symmetric weight $\mu_{x y}, x, y \in V$ such that $\mu_{x y}=\mu_{y x} \geq 0$. We say that $x$ and $y$ are neighbors, i.e., $\{x, y\} \in E$, denoted by $x \sim y$, if and only if $\mu_{x y}>0$.

Define $\mu(x)=\sum_{y \sim x} \mu_{x y}$, then it extends to a measure on $V$ by

$$
\mu(\Omega)=\sum_{x \in \Omega} \mu_{x}
$$

where $\Omega$ is a finite subset in $V$.

For $x, y \in V$, a path of length $n$ between $x$ and $y$ is a sequence $x_{0}, x_{1}, \cdots, x_{n}$ with $x_{0}=x, x_{n}=y$ and $x_{i-1} \sim x_{i}, 1 \leq i \leq n$. The induced graph distance $d(x, y)$ is the minimal number of edges in any path connecting $x$ and $y$. The graph $\Gamma$ is connected if $d(x, y)<\infty$ for all $x, y \in V$. Define balls in $\Gamma$ by

$$
B(x, r)=\{y \in V: d(x, y) \leq r, x \in V, r \geq 0\} .
$$

Any weighted graph $(\Gamma, \mu)$ admits a random walk on $\Gamma$ defined by the transition probabilities $p(x, y)=\frac{\mu_{x y}}{\mu(x)}$ if $x \sim y$ and otherwise 0 . Then $p$ is a reversible Markov kernel satisfying

$$
\mu(x) p(x, y)=\mu_{x y}=\mu(y) p(y, x), \quad \sum_{y} p(x, y)=1 .
$$

Denote by $C(V)=\{f: V \rightarrow \mathbb{R}\}$ and by $C_{0}(V)$ the set of functions in $C(V)$ with finite support. For $p \in[1, \infty)$, the $L^{p}$ norm of a function $f \in C(V)$ is given by

$$
\|f\|_{p}=\left(\sum_{x}|f(x)|^{p} \mu(x)\right)^{1 / p},
$$

The linear operator $P$ associated with the kernel $p$ is defined by

$$
P f(x)=\sum_{y} p(x, y) f(y) .
$$

We call the operator $I-P$ the (probabilistic) Laplacian on $\Gamma$.

The value of the discrete gradient on $\Gamma$ is defined by

$$
|\nabla f(x)|=\left(\frac{1}{2} \sum_{y} p(x, y)|f(x)-f(y)|^{2}\right)^{1 / 2} .
$$

Then we have that $\langle(I-P) f, f\rangle=\||\nabla f|\|_{2}^{2}$, where $\langle\cdot, \cdot\rangle$ denotes the inner product on $L^{2}(V, \mu)$.

Throughout this paper, we always assume that

- $\Gamma$ is locally finite, i.e., there exists $N \in \mathbb{N}$ such that any $x \in V$ has at most $N$ neighbors;

- $\mu(x) \geq c_{1}$ and $(\Gamma, \mu)$ has controlled weights, i.e., there exists $c_{2}>0$ such that for all $x, y \in V$

$$
x \sim y \text { implies } p(x, y) \geq c_{2} ;
$$

- The volume growth of $(\Gamma, \mu)$ is uniformly polynomial. That is, there exists $D>0$ such that $\mu(B(x, n)) \simeq n^{D}$.

Note that under these assumptions, we have that $\mu_{x y}>c$ and for $1 \leq p<\infty$,

$$
\sum_{x}|\nabla f(x)|^{p} \mu(x) \simeq \sum_{x, y}|f(x)-f(y)|^{p} \mu_{x y},
$$

see, for example, [1, p. 313] for a proof.

Our main results are as follows. 
Theorem 1.1. Let $(\Gamma, \mu)$ be a locally uniformly finite weighted graph with controlled weight. Assume that $\mu(B(x, n)) \simeq n^{D}, \forall n \in \mathbb{N}$. Then for $p \geq 1$, it holds that

$\left(P_{p}^{D}\right) \quad\left\|f-f_{n}\right\|_{L^{p}(B(x, n))} \leq C n^{\frac{D}{p}+\frac{1}{p^{\prime}}}\||\nabla f|\|_{L^{p}(B(x, 2 n))}$.

Theorem 1.2. Let $(\Gamma, \mu)$ be a locally uniformly finite weighted graph with controlled weight and let $\Omega \subset \Gamma$ be a bounded subset. Assume that $\mu(B(x, n)) \simeq n^{D}, \forall n \in \mathbb{N}$. Then for $p \geq 1$, it holds

$\left(S_{p}^{D}\right) \quad\|f\|_{p} \leq C \mu(\Omega)^{\frac{1}{p}+\frac{1}{p^{\prime} D}}\||\nabla f|\|_{p}, \quad \forall f \in C_{0}(\Omega)$.

Note that when $p=1$, the above inequality $\left(S_{p}^{D}\right)$ is trivial since it is equivalent to $\mu(\partial \Omega) \geq C$ following the co-area formula (see for instance [8, Proposition 2.3]).

Define the escape time $T(x, r)$ to be the mean exit time of a simple random walk on $\Gamma$ starting at $x$ from the ball with center $x$ and radius $r$. We say $\Gamma$ has escape time exponent $\beta>0$ if $T(x, r) \simeq r^{\beta}$ for $r \geq 1$. Well known estimates for random walks on graphs imply that $D \geq 1$ and $2 \leq \beta \leq D+1$. In particular, Vicsek graphs are borderline examples with $\beta=D+1$ (see [3]), which are of particular interest to us. In fact, we have

Proposition 1.3. Let $\Gamma$ be a Vicsek graph. Then the Poincaré inequality $\left(P_{p}^{D}\right)$ and Sobolev type inequality $\left(S_{p}^{D}\right)$ in Theorems 1.1 and 1.2 are optimal for $1 \leq p \leq \infty$.

Remark 1.4. In Theorem 1.1 and Theorem 1.2, we have variant parameters in inequalities $\left(P_{p}^{D}\right)$ and $\left(S_{p}^{D}\right)$, which depends on $p$ and $D$. When these inequalities are optimal, then the related parameters can't be improved to smaller numbers.

The paper is organized as follows. In Section 2, we give the proof of Theorem 1.1. Section 3 provides two different proofs of Theorem 1.2. Finally, we study the special case of Vicsek graphs and prove Proposition 1.3 .

\section{PoINCARÉ TYPe INEQUalities}

In this section, we will prove Theorem 1.1. Recall that under assumptions of volume growth, the $L^{1}$ and $L^{2}$-Poincare type inequalities were partially obtained in [11]. In fact, if the the volume growth is uniformly polynomial, i.e., $\mu(B(x, n)) \simeq n^{D}$, then for any $f \in C_{0}(V)$,

and

$$
\left\|f-f_{B(x, n)}\right\|_{L^{1}(B(x, n))} \leq C n^{D}\||\nabla f|\|_{L^{1}(B(x, 2 n))}, \quad \forall x \in V,
$$

$$
\left\|f-f_{B(x, n)}\right\|_{L^{2}(B(x, n))} \leq C n^{\frac{D+1}{2}}\||\nabla f|\|_{L^{2}(B(x, 2 n))}, \quad \forall x \in V .
$$

We will extend ideas from [11] (see also [12]) to prove our first result.

Proof of Theorem 1.1. For any $y, z \in B(x, n)$, we choose $\gamma_{y, z}$ as one of the shortest paths from $y$ to $z$. Define $\Gamma_{x, n}:=\left\{\gamma_{y, z}: y, z \in B(x, n)\right\}$. Let $e$ be an oriented edge in a path from $e_{-}$to $e_{+}$and $\mu_{e}=\mu_{e_{-} e_{+}}$.

Since

$$
|f(y)-f(z)| \leq \sum_{e \in \gamma_{y, z}}\left|f\left(e_{+}\right)-f\left(e_{-}\right)\right|,
$$

for $p=1$, we have

$$
\begin{aligned}
\mu(B(x, n)) \sum_{y \in B(x, n)}\left|f(y)-f_{B(x, n)}\right| \mu(y) & \leq \sum_{y, z \in B(x, n)}|f(y)-f(z)| \mu(y) \mu(z) \\
& \leq \sum_{y, z \in B(x, n)} \sum_{e \in \gamma_{y, z}}\left|f\left(e_{+}\right)-f\left(e_{-}\right)\right| \frac{\mu_{e}}{\mu_{e}} \mu(y) \mu(z)
\end{aligned}
$$




$$
\leq C \sum_{y, z \in B(x, n)}\left(\sum_{e \in \gamma_{y, z}}\left|f\left(e_{+}\right)-f\left(e_{-}\right)\right| \mu_{e}\right) \mu(y) \mu(z) .
$$

In the last inequality, $C$ depends on the weight $\mu$.

For $p>1$, the Hölder inequality leads to

$$
\begin{aligned}
\mu(B(x, n)) \sum_{y \in B(x, n)}\left|f(y)-f_{B(x, n)}\right|^{p} \mu(y) & \leq \mu(B(x, n))^{1-p} \sum_{y \in B(x, n)}\left|\sum_{z \in B(x, n)}[f(y)-f(z)] \mu(z)^{\frac{1}{p}+\frac{1}{p^{\prime}}}\right|^{p} \mu(y) \\
& \leq \mu(B(x, n))^{1-p} \sum_{y \in B(x, n)}\left(\sum_{z \in B(x, n)}|f(y)-f(z)|^{p} \mu(z)\right) \cdot\left(\sum_{z \in B(x, n)} \mu(z)\right)^{p-1} \mu(y) \\
& =\sum_{y, z \in B(x, n)}|f(y)-f(z)|^{p} \mu(y) \mu(z) \\
& \leq \sum_{y, z \in B(x, n)}\left(\sum_{e \in \gamma_{y, z}}\left|f\left(e_{+}\right)-f\left(e_{-}\right)\right| \frac{\mu_{e}^{1 / p}}{\mu_{e}^{1 / p}}\right)^{p} \mu(y) \mu(z) \\
& \leq \sum_{y, z \in B(x, n)}\left(\sum_{e \in \gamma_{y, z}}\left|f\left(e_{+}\right)-f\left(e_{-}\right)\right|^{p} \mu_{e}\right) \cdot\left(\sum_{e \in \gamma_{y, z}} \mu_{e}^{-1 /(p-1)}\right)^{p-1} \mu(y) \mu(z) .
\end{aligned}
$$

Since $\Gamma$ has controlled weights, the last term is bounded from above by

$$
C \sum_{e \in B(x, 2 n)}\left|f\left(e_{+}\right)-f\left(e_{-}\right)\right|^{p} \mu_{e} \cdot \sum_{y, z \in B(x, n)}\left|\gamma_{y, z}\right|^{p-1} \mu(y) \mu(z) .
$$

Take $K(x, n)=\sum_{y, z \in B(x, n)}\left|\gamma_{y, z}\right|^{p-1} \mu(y) \mu(z)$ for $p \geq 1$, then we get from (1.6)

$$
\left\|f-f_{B(x, n)}\right\|_{L^{p}(B(x, n))}^{p} \leq C K(x, n)\||\nabla f|\|_{L^{p}(B(x, 2 n))}^{p}, \forall p \geq 1 .
$$

One can see that $K(x, n) \leq(2 n)^{p-1} V(x, n)^{2}$. Therefore,

$$
\left\|f-f_{B(x, n)}\right\|_{L^{p}(B(x, n))}^{p} \leq C \mu(B(x, n))^{1+\frac{p-1}{D}}\||\nabla f|\|_{L^{p}(B(x, 2 n))}^{p} .
$$

Since $\mu(B(x, n)) \simeq n^{D}$, we have

$$
\left\|f-f_{B(x, n)}\right\|_{L^{p}(B(x, n))}^{p} \leq C n^{D+p-1}\||\nabla f|\|_{L^{p}(B(x, 2 n))}^{p} .
$$

This leads to $\left(P_{p}^{D}\right)$ and hence we finish the proof.

The pseudo-Poincaré type inequality follows now as a corollary.

Corollary 2.1. Let $(\Gamma, \mu)$ be a locally uniformly finite weighted graph with controlled weight. Assume that $\mu(B(x, n)) \simeq n^{D}, \forall n \in \mathbb{N}$. Then for $p \geq 1$, it holds that

$$
\left(P P_{p}^{D}\right) \quad\left\|f-f_{n}\right\|_{p} \leq C n^{\frac{D}{p}+\frac{1}{p^{\prime}}}\||\nabla f|\|_{p} .
$$

The proof is the similar to the one that Poincare inequality together with the volume doubling property implies the pseudo-Poincaré inequality, see for instance [14, Lemma 5.3.2]. We leave details to the interested reader. 


\section{SoboleV TYPE INEQUALITIES}

In this section, we will prove Theorem 1.2, for which we provide two different approaches. One is to use the Poincaré type inequality $\left(P P_{p}^{D}\right)$, following [6, Section III] (see also [8, Proposition 2.6]).

Proof of Theorem 1.2. We first treat the case $p>1$. Note that for any $f \geq 0$ in $C_{0}(\Omega)$, there holds for any $x \in V$ and $n \geq 1$

$$
\left|f_{n}(x)\right|=\left|\frac{1}{\mu(B(x, n))} \sum_{y \in B(x, n)} f(y) \mu(y)\right| \leq C_{D} n^{-D}\|f\|_{1} .
$$

Write

$$
\|f\|_{p}^{p}=\left(f-f_{n}, f^{p-1}\right)+\left(f_{n}, f^{p-1}\right) .
$$

It follows from Hölder inequalities, $\left(P P_{p}^{D}\right)$, and (3.1) that

$$
\begin{aligned}
\|f\|_{p}^{p} & \leq\left\|f-f_{n}\right\|_{p} \cdot\|f\|_{p}^{p-1}+\left\|f_{n}\right\|_{\infty} \cdot\left\|f^{p-1}\right\|_{1} \\
& \leq C n^{\frac{D}{p}+\frac{1}{p^{\prime}}}\||\nabla f|\|_{p} \cdot\|f\|_{p}^{p-1}+\frac{C_{D} \mu(\Omega)}{n^{D}}\|f\|_{p}^{p} .
\end{aligned}
$$

Choosing $n$ to be the integer such that $n^{D} \simeq 2 C_{D} \mu(\Omega)$, then

$$
\|f\|_{p} \leq C \mu(\Omega)^{\frac{1}{p}+\frac{1}{p^{\prime} D}}\||\nabla f|\|_{p} .
$$

The inequality also holds for any $f \in C_{0}(\Omega)$.

Next let $p=1$. For any $\lambda>0$, write

$$
\mu(\{|f| \geq \lambda\}) \leq \mu\left(\left\{\left|f-f_{n}\right|>\lambda / 2\right\}\right)+\mu\left(\left\{\left|f_{n}\right|>\lambda / 2\right\}\right) .
$$

Taking $n \simeq\left(2\|f\|_{1} / \lambda\right)^{1 / D}$ to be the integer such that $\left\{\left|f_{n}\right|>\lambda / 2\right\}=\emptyset$. Therefore by $\left(P P_{1}^{D}\right)$,

$$
\mu(\{|f| \geq \lambda\}) \leq \frac{2}{\lambda}\left\|f-f_{n}\right\|_{1} \leq \frac{2}{\lambda} n^{D}\||\nabla f|\|_{1} \leq C\|f\|_{1}\||\nabla f|\|_{1} .
$$

Taking $\lambda=1$ and $f=\mathbf{1}_{\Omega}$ yields $\mu(\partial \Omega) \geq C$, which is equivalent to $\left(S_{1}^{D}\right)$.

The second approach, without using the Poincaré inequality, is inspired by the Faber-Krahn inequality in [2]. That is, for a graph $(\Gamma, \mu)$ with controlled weights, the Faber-Krahn inequality as follows holds for any non-empty finite set $\Omega \subset \Gamma$,

$$
\lambda_{1}(\Omega):=\inf _{f \in \mathcal{C}_{0}(\Omega)} \frac{\sum_{x \in \Omega}|\nabla f(x)|^{2} \mu(x)}{\sum_{x \in \Omega}|f(x)|^{2} \mu(x)} \geq \frac{c}{r(\Omega) \mu(\Omega)},
$$

where

$$
r(\Omega)=\max \{r \in \mathbb{N}: \exists x \in \Omega \text { such that } B(x, r) \subset \Omega\} .
$$

If $\Gamma$ has polynomial volume upper bound $\mu(B(x, r)) \gtrsim r^{D}$, the Faber-Krahn inequality is also the $L^{2}$-Sobolev type inequality in our theorme. Moreover, this inequality is sharp at all scales of the volume on Vicsek graphs ([2, Theorem 4.1]).

An alternative proof of Theorem 1.2. For any function $f \in C_{0}(\Omega)$, assume $\max _{x \in \Omega}|f(x)|=1$ (otherwise we can normalize $f$ ). Then we have

$$
\sum_{x \in \Omega}|f(x)|^{p} \mu(x) \leq \mu(\Omega)
$$

Consider a point $x_{0}$ such that $\left|f\left(x_{0}\right)\right|=1$ and the largest integer $n$ such that the ball $B\left(x_{0}, n\right) \subset \Omega$. Obviously we have $n \leq r(\Omega)$. Also, there exists a sequence of points

$$
x_{0} \sim x_{1} \sim x_{2} \sim \cdots \sim x_{n} \sim x_{n+1}
$$


starting from $x_{0}$ and terminating at a point $x_{n+1} \notin \Omega$.

Therefore, by (1.6) and the Hölder inequality

$$
\begin{aligned}
\sum_{x \in \Omega}|\nabla f(x)|^{p} \mu(x) & \simeq \sum_{x, y \in \Omega}|f(x)-f(y)|^{p} \mu_{x y} \\
& \geq \frac{c}{n^{p-1}}\left(\sum_{i=0}^{n}\left|f\left(x_{i}\right)-f\left(x_{i+1}\right)\right|\right)^{p} \geq \frac{c}{n^{p-1}},
\end{aligned}
$$

where the last inequality is due to the fact that

$$
\sum_{i=0}^{n}\left|f\left(x_{i}\right)-f\left(x_{i+1}\right)\right| \geq\left|f\left(x_{0}\right)-f\left(x_{n+1}\right)\right|=1 .
$$

It follows from (3.2) and (3.3) that

$$
\frac{\sum_{x \in \Omega}|\nabla f(x)|^{p} \mu(x)}{\sum_{x \in \Omega}|f(x)|^{p} \mu(x)} \geq \frac{c}{n^{p-1} \mu(\Omega)} \geq \frac{c}{\mu(\Omega)^{1+(p-1) / D}} .
$$

Eventually, we obtain $\left(S_{p}^{D}\right)$ and the proof is completed.

\section{Optimality ON Vicsek GRAPHS}

Considering the Vicsek graphs, we assume that the weight $\mu$ is the standard weight. Recall the construction of a Vicsek graph $\Gamma$ on $\mathbb{R}^{d}$ (taken from [9]): Let $Q_{r}$ denote the cube in $\mathbb{R}^{n}$

$$
Q_{r}=\left\{x \in \mathbb{R}^{d}: 0 \leq x_{i} \leq r, i=1,2, \cdots, d\right\} .
$$

Construct an increasing sequence $\left\{\Gamma_{k}\right\}$ of finite graphs as subsets of $Q_{3^{k}}$. Let $\Gamma_{1}$ be the set of $2^{d}+1$ points containing all vertices of $Q_{1}$ and the center of $Q_{1}$. Define $2^{d}$ edges in $\Gamma_{1}$ as segments connecting the center with the corners. Assuming that $\Gamma_{k}$ is already constructed, define $\Gamma_{k+1}$ as follows. The cube $Q_{3^{k}+1}$ is naturally divided into $3^{n}$ congruent copies of $Q_{3^{k}}$; select $2^{d}+1$ of the copies of $Q_{3^{k}}$ by taking the corner cubes and the center one. In each of the selected copies of $Q_{3^{k}}$ construct a congruent copy of graph $\Gamma_{k}$, and define $\Gamma_{k+1}$ as the union of all $2^{d}+1$ copies of $\Gamma_{k}$ (merged at the corners). Then the Vicsek tree $\Gamma$ is the union of all $\Gamma_{k}$, $k \geq 1$ (see Figure 1). Consider the natural weight $\mu_{x y}=1$ if $x \sim y$ and otherwise 0 . For all $x \in \Gamma, r \geq 1$ and $k>0, \Gamma$ satisfies

and

$$
\mu(B(x, r)) \simeq r^{D}
$$

$$
p_{k}(x, x) \simeq C k^{-\frac{D}{D+1}} .
$$

where $D=\log _{3}\left(2^{d}+1\right)$. By Theorem 1.1, the Vicsek graph satisfies the following scale-invariant Poincaré inequality: for any $f \in \mathcal{C}_{0}(\Gamma)$,

$$
\left\|f-f_{B(x, r)}\right\|_{L^{2}(B(x, n))} \leq C r^{\frac{D+1}{2}}\||\nabla f|\|_{L^{2}(B(x, 2 r))}, \quad \forall x \in V,
$$

Since $D>1$, this inequality is strictly weaker than $\left(P_{2}\right)$.

Proof of Proposition 1.3. It suffices to show the optimality of the generalized Sobolev inequality $\left(S_{p}^{D}\right)$ for $p \geq 1$. Indeed, note that we can obtain $\left(S_{p}^{D}\right)$ from $\left(P_{p}^{D}\right)$ in the proof of Theorem 1.2. If the exponent $D(p)=\frac{D}{p}+\frac{1}{p^{\prime}}$ of $r$ in $\left(P_{p}^{D}\right)$ is not optimal, then $\left(S_{p}^{D}\right)$ can also be improved, which contradicts its optimality.

In fact, with the same functions and subsets, we can also show the optimality of $\left(S_{p}^{D}\right)$ for $p \geq 1$. 

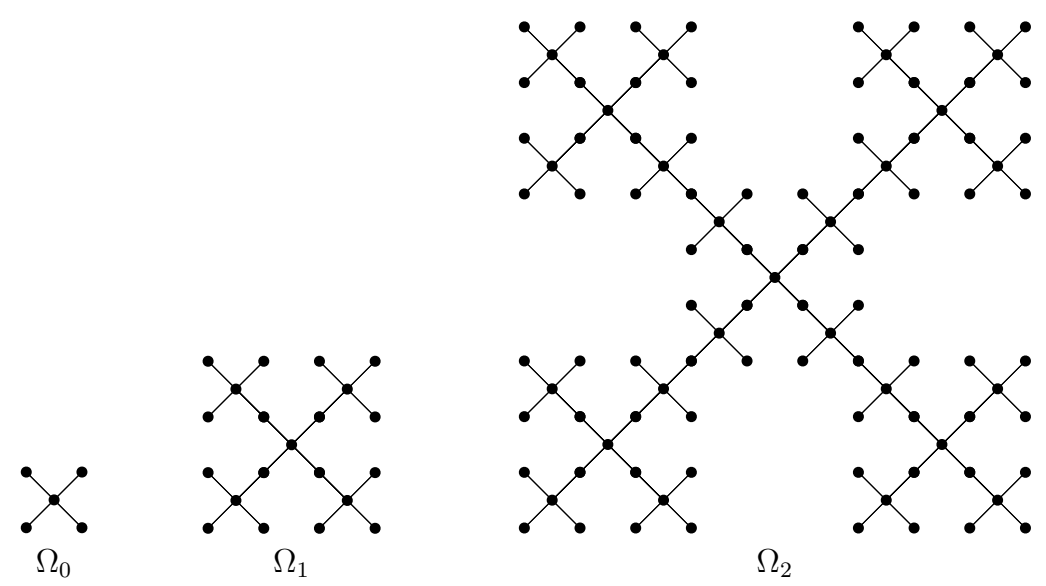

FigURE 1. The first steps of the Vicsek graph with parameter $D=\log _{3} 5$ built in $\mathbb{R}^{2}$.

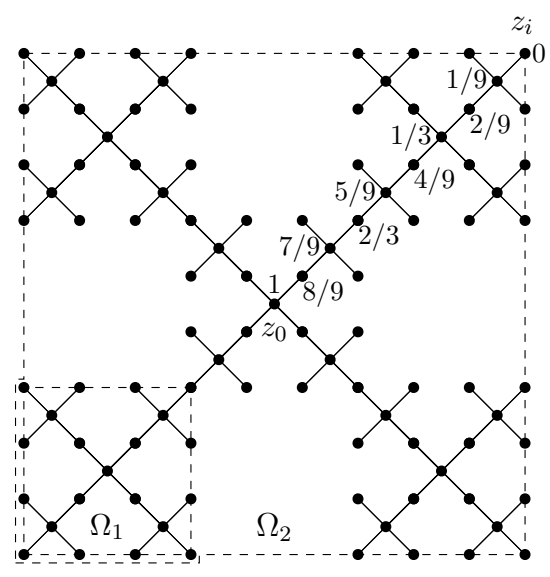

FIGURE 2. the function $g_{2}$ on the diagonal $z_{0} z_{i}$

Let $\Omega_{n}=\Gamma \bigcap\left[0,3^{n}\right]^{d}$ be the same subset as in [2], where $q=2^{d}+1=3^{D}$. Hence $\mu\left(\Omega_{n}\right) \simeq q^{n}$. Denote by $z_{0}$ the centre of $\Omega_{n}$ and by $z_{i}, i \geq 1$ its corners. Define $F_{n}$ as follows: $F_{n}\left(z_{0}\right)=1, F_{n}\left(z_{i}\right)=0, i \geq 1$, and extend $F_{n}$ as a harmonic function in the rest of $\Omega_{n}$. Then $F_{n}$ is linear on each of the paths of length $3^{n}$, which connects $z_{0}$ with the corners $z_{i}$, and is constant elsewhere. More exactly, if $z$ belongs to some $\gamma_{z_{0}, z_{i}}$, then $F_{n}(z)=3^{-n} d\left(z_{i}, z\right)$. If not, then $F_{n}(z)=F_{n}\left(z^{\prime}\right)$, where $z^{\prime}$ is the nearest vertex in certain line of $z_{0}$ and $z_{i}$. See Figure 2, which we take from [2].

For any $x$ in the $n-1$ block with centre $z_{0}$, we have $F_{n}(x) \geq \frac{2}{3}$. Therefore

$$
\sum_{x \in \Omega_{n}}\left|F_{n}(x)\right|^{p} \mu(x) \geq(2 / 3)^{p} \mu\left(\Omega_{n-1}\right) \simeq \mu\left(\Omega_{n}\right) .
$$


Also, since $\left|F_{n}(x)-F_{n}(y)\right|=3^{-n}$ for any two neighbours $x, y$ on each of the diagonals connecting $z_{0}$ and $z_{i}$, and otherwise $F_{n}(x)-F_{n}(y)=0$, we obtain

$$
\begin{aligned}
\left\|\left|\nabla F_{n}\right|\right\|_{p}^{p} & \simeq \sum_{x, y \in \Omega_{n}}\left|F_{n}(x)-F_{n}(y)\right|^{p} \mu_{x y} \leq \sum_{i=1}^{2^{N}} 3^{-n p} d\left(z_{0}, z_{i}\right)=2^{N} 3^{-n(p-1)} \\
& \simeq \mu\left(\Omega_{n}\right)^{-\frac{p-1}{D}} .
\end{aligned}
$$

Finally combining the above two estimates, we have

$$
\frac{\left\|\left|\nabla F_{n}\right|\right\|_{p}}{\left\|F_{n}\right\|_{p}} \lesssim \mu\left(\Omega_{n}\right)^{-\frac{1}{p^{\prime} D}-\frac{1}{p}} .
$$

This finishes the proof.

Remark 4.1. In [5], it was proved that $\left\|(I-P)^{1 / 2} f\right\|_{p} \leq C\||\nabla f|\|_{p}$ doesn't hold for $1<p<2$ and hence by duality the Riesz transform $\nabla(I-P)^{-1 / 2}$ is not bounded on $L^{p}$ for $p>2$. This result is strikingly different from Euclidean spaces and its proof relies on an argument of contradiction for which we used the same family of functions as in the above proof. It would be of further interest to explore the intrinsic role that the weaker Poincaré inequalities play on the boundedness of the Riesz transform.

\section{REFERENCES}

[1] N. Badr and E. Russ. Interpolation of Sobolev spaces, Littlewood-Paley inequalities and Riesz transforms on graphs. Publ. Mat., 53(2):273-328, 2009. 2

[2] M. Barlow, T. Coulhon, and A. Grigor'yan. Manifolds and graphs with slow heat kernel decay. Invent. Math., 144(3):609-649, 2001. $1,5,7$

[3] M. T. Barlow. Which values of the volume growth and escape time exponent are possible for a graph? Rev. Mat. Iberoamericana, 20(1):1-31, 2004. 1, 3

[4] M. T. Barlow. Random walks and heat kernels on graphs, volume 438 of London Mathematical Society Lecture Note Series. Cambridge University Press, Cambridge, 2017. 1, 2

[5] Li Chen, Thierry Coulhon, Joseph Feneuil, and Emmanuel Russ. Riesz transform for $1 \leq p \leq 2$ without Gaussian heat kernel bound. J. Geom. Anal., 27(2):1489-1514, 2017. 8

[6] T. Coulhon. Dimensions at infinity for Riemannian manifolds. Potential Anal., 4(4):335-344, 1995. Potential theory and degenerate partial differential operators (Parma). 1, 5

[7] T. Coulhon. Espaces de Lipschitz et inégalités de Poincaré. J. Funct. Anal., 136(1):81-113, 1996. 1

[8] T. Coulhon. Heat kernel and isoperimetry on non-compact Riemannian manifolds. In Heat kernels and analysis on manifolds, graphs, and metric spaces (Paris, 2002), volume 338 of Contemp. Math., pages 65-99. Amer. Math. Soc., Providence, RI, 2003. $1,3,5$

[9] T. Coulhon and A. Grigor'yan. Pointwise estimates for transition probabilities of random walks on infinite graphs. In Fractals in Graz 2001, Trends Math., pages 119-134. Birkhäuser, Basel, 2003. 6

[10] T. Coulhon, I. Holopainen, and L. Saloff-Coste. Harnack inequality and hyperbolicity for subelliptic $p$-Laplacians with applications to Picard type theorems. Geom. Funct. Anal., 11(6):1139-1191, 2001. 1

[11] T. Coulhon and L. Saloff-Coste. Isopérimétrie pour les groupes et les variétés. Rev. Mat. Iberoamericana, 9(2):293-314, 1993. 1, 3

[12] P. Diaconis and D. Stroock. Geometric bounds for eigenvalues of Markov chains. Ann. Appl. Probab., 1(1):36-61, 1991. 3

[13] L. Saloff-Coste. A note on Poincaré, Sobolev, and Harnack inequalities. Internat. Math. Res. Notices, (2):27-38, 1992. 1

[14] L. Saloff-Coste. Aspects of Sobolev-type inequalities, volume 289 of London Mathematical Society Lecture Note Series. Cambridge University Press, Cambridge, 2002. 1, 4

[15] Laurent Saloff-Coste. Sobolev inequalities in familiar and unfamiliar settings. In Sobolev spaces in mathematics. I, volume 8 of Int. Math. Ser. (N. Y.), pages 299-343. Springer, New York, 2009. 1

Department of Mathematics, University of Connecticut, Storrs, CT 06269 